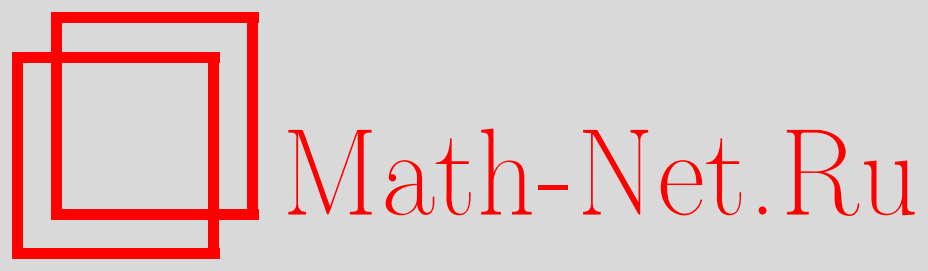

Д. В. Миллионщиков, Алгебра формальных векторных полей на прямой и гипотеза Бухштабера, Функи. анализ и его прил., 2009, том 43, выпуск 4, 26-44

DOI: https://doi.org/10.4213/faa2967

Использование Общероссийского математического портала Math$\mathrm{Net.Ru}$ подразумевает, что вы прочитали и согласны с пользовательским соглашением

http://www. mathnet.ru/rus/agreement

Параметры загрузки:

IP : 18.209 .158 .208

26 апреля 2023 г., $16: 28: 45$

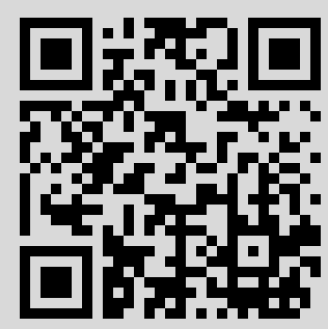




\title{
Алгебра формальных векторных полей на прямой и гипотеза Бухштабера*
}

\author{
(c) 2009. Д. В. Миллионщиков
}

\begin{abstract}
Рассматривается алгебра Ли $L_{1}$ формальных векторных полей на прямой, обращающихся в нуль вместе с первой производной в начале координат. В. М. Бухштабер и А. В. Шокуров показали, что универсальная обертывающая алгебра $U\left(L_{1}\right)$ изоморфна алгебре Ландвебера-Новикова $S$, тензорно умноженной на вещественные числа. Когомологии $H^{*}\left(L_{1}, \mathbb{R}\right)=H^{*}\left(U\left(L_{1}\right)\right)$ были первоначально вычислены Л. В. Гончаровой. Из ее вычислений следует, что умножение в когомологиях $H^{*}\left(L_{1}, \mathbb{R}\right)$ тривиально. Бухштабер высказал гипотезу, что когомологии $H^{*}\left(L_{1}\right)$ порождаются одномерными коциклами с помощью нетривиальных произведений Масси. Б. Л. Фейгин, Д. Б. Фукс и В. С. Ретах нашли выражение аддитивных образующих $H^{*}\left(L_{1}\right)$ в требуемом виде, но указанные ими прозведения Масси, как выяснилось позднее, содержат нулевой элемент. В настоящей статье мы доказываем, что $H^{*}\left(L_{1}\right)$ рекуррентно порождается с помощью нетривиальных произведений Масси двумя одномерными коциклами из $H^{1}\left(L_{1}\right)$.
\end{abstract}

\section{Предисловие}

В конце 70-х годов Бухштабер и Шокуров [7] открыли, что алгебра Ландвебера-Новикова $S$, возникающая в теории комплексных кобордизмов, тензорно умноженная на поле вещественных чисел, $S \otimes \mathbb{R}$, изоморфна универсальной обертывающей алгебре $U\left(L_{1}\right)$ алгебры Ли $L_{1}$ формальных векторных полей на вещественной прямой $\mathbb{R}$, обращающихся в нуль в начале координат вместе с первой производной. Еще раньше, в 1970 г., Бухштабер [6] построил триградуированную спектральную последовательность для вычисления члена $E_{2}$ спектральной последовательности Адамса-Новикова. Ключевую роль в его конструкции играли целочисленные когомологии алгебры $S$. В том же 1970 г. Гельфанд поставил проблему вычисления когомологий нильпотентных подалгебр $L_{k}$ алгебры $L_{1}$ [8]. Отметим, что при $k=1$ эта задача, успешно решенная Гончаровой [9] тремя годами позднее, в работе [7] формулируется как вычисление рациональных когомологий $H^{*}(S, \mathbb{Q})$ алгебры Ландвебера-Новикова. С другой стороны, результат [7] приводит к целочисленному аналогу данной задачи, как это было отмечено в [14]. Нетрудно видеть, что $H^{1}(S, \mathbb{Z})=\mathbb{Z} \oplus \mathbb{Z}$. Группа же $H^{2}(S, \mathbb{Z})$, вычисленная в [7], имеет большую конечную подгруппу. Вопрос о полном вычислении когомологий $H^{*}(S, \mathbb{Z})$ является по-прежнему открытым.

Из теоремы Гончаровой [9] вытекает, что в алгебре когомологий $H^{*}\left(L_{1}\right)$ умножение тривиально. Бухштабер высказал гипотезу, что когомологии $H^{*}\left(L_{1}\right)$ порождаются одномерными когомологиями $H^{1}\left(L_{1}\right)$ с помощью нетривиальных произведений Масси. Фейгин, Фукс и Ретах [13] представили базисные коциклы из $H^{*}\left(L_{1}\right)$ в виде произведений Масси. Но все произведения, рассмотренные

*Частично поддержано грантами РФФИ 08-01-00541, 08-01-91855 и грантом Президента РФ поддержки ведущих научных школ №2185.2008.1. 
ими, являются тривиальными, т. е. содержат 0. Позднее Артельных [1] нашел выражения с помощью нетривиальных произведений Масси для части базисных коциклов из $H^{*}\left(L_{1}\right)$, но его короткая заметка не содержит полных доказательств.

В настоящей работе мы доказываем гипотезу Бухштабера в ее первоначальной формулировке. Основным результатом является теорема 3.1 , утверждающая, что алгебра когомологий $H^{*}\left(L_{1}\right)$ порождается при помощи нетривиальныx произведений Масси двумя базисными коциклами из $H^{1}\left(L_{1}\right)$. В ее доказательстве существенную роль играет техника, развитая Фейгиным, Фуксом и Ретахом в [13], а также формула Бенуа-Сент-Обана [5] для особых векторов модулей Верма над алгеброй Вирасоро.

Автор искренне благодарен Л. А. Алания, И. К. Бабенко и В. М. Бухштаберу за внимание к работе и многочисленные ценные замечания.

\section{§1. Когомологии положительной части алгебры Витта}

Пусть $\mathfrak{g}$ - алгебра Ли над полем $\mathbb{K}=\mathbb{R}$ или $\mathbb{C}$ и $\rho: \mathfrak{g} \rightarrow \mathfrak{g l}(V)$ - ее линейное представление ( $V$ является $\mathfrak{g}$-модулем). В дальнейшем обозначим через $C^{q}(\mathfrak{g}, V)$ пространство $q$-линейных (непрерывных) кососимметрических отображений из $\mathfrak{g}$ в $V$. Можно определить алгебраический комплекс $\left(C^{*}(\mathfrak{g}, V), d\right)$ :

$$
V \stackrel{d_{0}}{\longrightarrow} C^{1}(\mathfrak{g}, V) \stackrel{d_{1}}{\longrightarrow} C^{2}(\mathfrak{g}, V) \stackrel{d_{2}}{\longrightarrow} \cdots \stackrel{d_{q-1}}{\longrightarrow} C^{q}(\mathfrak{g}, V) \stackrel{d_{q}}{\longrightarrow} \cdots,
$$

где дифференциал $d_{q}$ определен следующим образом:

$$
\begin{aligned}
\left(d_{q} f\right)\left(X_{1}, \ldots, X_{q+1}\right) & =\sum_{i=1}^{q+1}(-1)^{i+1} \rho\left(X_{i}\right)\left(f\left(X_{1}, \ldots, \widehat{X}_{i}, \ldots, X_{q+1}\right)\right) \\
& +\sum_{1 \leqslant i<j \leqslant q+1}(-1)^{i+j-1} f\left(\left[X_{i}, X_{j}\right], X_{1}, \ldots, \widehat{X}_{i}, \ldots, \widehat{X}_{j}, \ldots, X_{q+1}\right) .
\end{aligned}
$$

Когомологии этого комплекса называются когомологиями алгебры Ли $\mathfrak{g}$ с коэффициентами в представлении $\rho: \mathfrak{g} \rightarrow \mathfrak{g l}(V)$. В частности, когомологии комплекса $\left(C^{*}(\mathfrak{g}, \mathbb{K}), d\right)$ с коэффициентами в тривиальном представлении $\rho: \mathfrak{g} \rightarrow$ $\mathfrak{g l}(\mathbb{K})$ называются когомологиями с тривиальными коэффициентами алгебры Ли $\mathfrak{g}$ и обозначаются через $H^{*}(\mathfrak{g})$.

Алгебра Витта $W$ есть линейная оболочка следующей системы дифференциальных операторов (векторных полей) на вещественной прямой $\mathbb{R}^{1}$ с фиксированной координатой $x$ :

$$
e_{i}=x^{i+1} \frac{d}{d x}, \quad i \in \mathbb{Z}, \quad\left[e_{i}, e_{j}\right]=(j-i) e_{i+j} \quad \forall i, j \in \mathbb{Z} .
$$

Алгебру Ли формальных рядов $\sum_{i \geqslant 1}^{+\infty} a_{i} e_{i}$ с коммутатором [ , ], определенным на базисных векторах $e_{i}$ по формуле (2), следуя [9], [14], будем обозначать через $L_{1}$ и называть алгеброй формальных векторных полей на прямой $\mathbb{R}^{1}$, обращающихся в нуль в начале координат вместе с первой производной.

Возьмем двойственный базис $e^{1}, \ldots, e^{n}, \ldots$ в $L_{1}^{*}$. Рассмотрим вторую градуировку комплекса $C^{*}\left(L_{1}, \mathbb{K}\right)$ :

$$
C^{*}\left(L_{1}, \mathbb{K}\right)=\bigoplus_{\lambda=1}^{\infty} C_{\lambda}^{*}\left(L_{1}, \mathbb{K}\right)
$$


где подпространство $C_{\lambda}^{q}\left(L_{1}, \mathbb{K}\right)$ порождено $q$-формами $\left\{e^{i_{1}} \wedge \cdots \wedge e^{i_{q}}, i_{1}+\cdots+i_{q}=\right.$ $\lambda\}$. Элементы подпространства $C_{\lambda}^{q}\left(L_{1}, \mathbb{K}\right)$ будут называться элементами веса $\lambda$. Например, моном $e^{i_{1}} \wedge \cdots \wedge e^{i_{q}}$ имеет степень $q$ и вес $\lambda=i_{1}+\cdots+i_{q}$.

Эта градуировка совместима с внешним умножением форм. Последнее индуцирует структуру биградуированной алгебры в когомологиях $H^{*}\left(L_{1}, \mathbb{R}\right)$ :

$$
H_{k}^{q}\left(L_{1}, \mathbb{R}\right) \otimes H_{l}^{p}\left(L_{1}, \mathbb{R}\right) \rightarrow H_{k+l}^{q+p}\left(L_{1}, \mathbb{R}\right) .
$$

Теорема 1.1 [9]. Пространство q-мерньх когомологий $H^{q}\left(L_{1}, \mathbb{R}\right)$ двумерно для любого $q \geqslant 1$, более того, оно является прямой суммой двух своих одномерных однородных подпространств:

$$
H^{q}\left(L_{1}, \mathbb{R}\right)=H_{\left(3 q^{2}-q\right) / 2}^{q}\left(L_{1}, \mathbb{R}\right) \oplus H_{\left(3 q^{2}+q\right) / 2}^{q}\left(L_{1}, \mathbb{R}\right) .
$$

В дальнейшем мы будем обозначать через $g_{ \pm}^{q}$ образующие подпространств $H_{\left(3 q^{2} \pm q\right) / 2}^{q}\left(L_{1}, \mathbb{R}\right)$ соответственно. Числа $e_{ \pm}(q)=\frac{1}{2}\left(3 q^{2} \pm q\right)$ называются пентагональными числами Эйлера. Легко проверить, что сумма двух чисел Эйлера $e_{ \pm}(q)+e_{ \pm}(l)$ не равняется $e_{ \pm}(q+l)$ ни для каких $q$ и $l$. Следовательно, в алгебре когомологий $H^{*}\left(L_{1}, \mathbb{R}\right)$ умножение тривиально.

Пример 1.2. $H^{1}\left(L_{1}, \mathbb{R}\right)$ линейно порождается элементами $g_{-}^{1}=\left[e^{1}\right]$ и $g_{+}^{1}=$ $\left[e^{2}\right]$, а базис пространства $H^{2}\left(L_{1}, \mathbb{R}\right)$ состоит из двух классов $g_{-}^{2}=\left[e^{1} \wedge e^{4}\right]$ и $g_{+}^{2}=\left[e^{2} \wedge e^{5}-3 e^{3} \wedge e^{4}\right]$ весов 5 и 7 соответственно. Это сразу видно из формулы для дифференциала $d: C^{1}\left(L_{1}, \mathbb{R}\right) \rightarrow C^{2}\left(L_{1}, \mathbb{R}\right)$, который является двойственным отображением к скобке Ли [ , ] алгебры $L_{1}$ :

$$
d e^{1}=d e^{2}=0, \quad d e^{n}=\sum_{i=1}^{[n / 2]}(n-2 i) e^{i} \wedge e^{n-i}, \quad n \geqslant 3 .
$$

Здесь и далее $[a]$ будет обозначать класс когомологий коцикла $a$ (не путать с целой частью $[x]$ вещественного числа $x)$; там, где это не будет вызывать путаницы, для упрощения записи произведений Масси мы будем опускать квадратные скобки у одномерных классов когомологий, например, $e^{1}$ будет иногда обозначать и коцикл, и соответствующий класс когомологии.

Пусть $V=\bigoplus_{i} V_{i}$ есть $\mathbb{Z}$-градуированный $L_{1}$-модуль с одномерными или тривиальными однородными компонентами $V_{i}$, заданный при помощи базиса $f_{i}: V_{i}=\left\langle f_{i}\right\rangle$. Комплекс $\left(C^{*}\left(L_{1}, V\right), d\right)$ имеет вторую градуировку $p$, которую мы также будем называть весом. Вес монома $e^{i_{1}} \wedge \cdots \wedge e^{i_{q}} \otimes f_{k} \in C^{q}\left(L_{1}, V\right)$ определен по правилу

$$
p\left(e^{i_{1}} \wedge \cdots \wedge e^{i_{q}} \otimes f_{k}\right)=k-i_{1}-\cdots-i_{q} .
$$

Эта градуировка совместима с дифференциалом $d$, и когомологии $H^{*}\left(L_{1}, \mathbb{R}\right)=$ $\bigoplus_{s} H_{s}^{*}\left(L_{1}, \mathbb{R}\right)$ также биградуированы.

Алгебра Вирасоро Vir является бесконечномерной алгеброй Ли, заданной своим базисом $\left\{z, e_{i}, i \in \mathbb{Z}\right\}$ и коммутационными соотношениями

$$
\left[e_{i}, z\right]=0 \quad \forall i \in \mathbb{Z}, \quad\left[e_{i}, e_{j}\right]=(j-i) e_{i+j}+\frac{j^{3}-j}{12} \delta_{-i, j} z .
$$

Vir является одномерным центральным расширением алгебры Витта $W$ (одномерный центр порождается элементом $z$ ). 
Замечание. В работах [10], [12], [13] символом $L_{1}$ обозначается «положительная» часть $W_{+}$алгебры Витта $W$ (алгебры Вирасоро Vir), т. е. алгебра полиномиальных векторных полей на прямой $\mathbb{R}$, обращающихся в нуль в начале координат вместе с первой своей производной. В дальнейшем мы будем использовать символ $L_{1}$ для обозначения обеих этих алгебр. Это не вызовет путаницы, так как когомологии $H^{*}\left(L_{1}\right)$ и $H^{*}\left(W_{+}\right)$изоморфны ([9], [14]).

\section{§2. Произведения Масси и теорема Ретаха-Фейгина-Фукса}

В этом параграфе мы следуем работе Бабенко и Тайманова [2], в которой был предложен подход с использованием так называемых формальных связностей для определения высших произведений Масси [18]. Пусть $\mathscr{A}=\bigoplus_{l \geqslant 0} \mathscr{A}^{l}$ - дифференциальная градуированная алгебра над полем $\mathbb{K}$. Это означает, что заданы следующие операции: ассоциативное произведение с единицей 1

$$
\wedge: \mathscr{A}^{l} \otimes \mathscr{A}^{m} \rightarrow \mathscr{A}^{l+m}, \quad l, m \geqslant 0, l, n \in \mathbb{Z},
$$

такое, что $a \wedge b=(-1)^{l m} b \wedge a$ для $a \in \mathscr{A}^{l}, b \in \mathscr{A}^{m}$, и дифференциал

$$
d: \mathscr{A}^{l} \rightarrow \mathscr{A}^{l+1}, \quad l \geqslant 0,
$$

удовлетворяющий правилу Лейбница $d(a \wedge b)=d a \wedge b+(-1)^{l} a \wedge d b\left(a \in \mathscr{A}^{l}\right)$ и условию $d^{2}=0$. Ли $\mathfrak{g}$.

Пример 2.1. $\mathscr{A}=C^{*}(\mathfrak{g}, \mathbb{K})-$ коцепной комплекс произвольной алгебры

Для данной дифференциальной градуированной алгебры $(\mathscr{A}, d)$ мы обозначим через $L T_{n}(\mathscr{A})$ пространство всех нижнетреугольных $(n+1) \times(n+1)$-матриц с матричными элементами из $\mathscr{A}$ и нулевой главной диагональю. Оно обладает структурой дифференциальной алгебры относительно обычного матричного умножения (матричные элементы перемножаются как элементы алгебры $\mathscr{A}$ ). Дифференциал $d$ в $L T_{n}(\mathscr{A})$ определен формулой

$$
d A=\left(d a_{i j}\right)_{1 \leqslant i, j \leqslant n+1}, \quad A=\left(a_{i j}\right)_{1 \leqslant i, j \leqslant n+1} .
$$

Инволюция $a \rightarrow \bar{a}=(-1)^{k+1} a, a \in A^{k}$, в алгебре $\mathscr{A}$ может быть продолжена до инволюции алгебры матриц $L T_{n}(\mathscr{A})$ следующим образом: $\bar{A}=\left(\bar{a}_{i j}\right)_{1 \leqslant i, j \leqslant n+1}$. Она обладает такими свойствами:

$$
\overline{\bar{A}}=A, \quad \overline{A B}=-\overline{A B}, \quad \overline{d A}=-d \bar{A} .
$$

Для дифференциала (3) также справедливо обобщенное правило Лейбница

$$
d(A B)=(d A) B-\bar{A}(d B) .
$$

В центре алгебры $L T_{n}(\mathscr{A})$ рассмотрим подпространство $I_{n}(\mathscr{A})$, состоящее из матриц следующего вида:

$$
\left(\begin{array}{ccc}
0 & \ldots & 0 \\
\vdots & \ddots & \vdots \\
\tau & \ldots & 0
\end{array}\right), \quad \tau \in \mathscr{A}
$$

Определение 2.2 [2]. Матрица $A \in L T_{n}(\mathscr{A})$ называется матрицей формальной связности, если она удовлетворяет обобщенному уравнению МаурераКартана

$$
\mu(A)=d A-\bar{A} \cdot A \in I_{n}(\mathscr{A}) .
$$


Предложение 2.3 [2]. Пусть $A$ - матрица формальной связности. Тогда элемент $\tau$ матрицы $\mu(A) \in I_{n}(\mathscr{A})$ является замкнутым: $d \tau=0$.

Определение 2.4. Коцикл $c(A)=-\tau+d a_{n+1,1}$ называется ассоциированным коциклом матрицы формальной связности $A$.

Определение 2.5. $n$-местное произведение $\left\langle a_{1}, \ldots, a_{n}\right\rangle$ определено, если существует по крайней мере одна матрица формальной связности $A$ с элементами $a_{n}, \ldots, a_{1}$ на второй диагонали. Если произведение определено, то $\left\langle a_{1}, \ldots, a_{n}\right\rangle$ состоит из всех когомологических классов $\alpha \in H^{*}(\mathscr{A})$, для которых существует матрица формальной связности $A$, такая, что $c(A)$ представляет $\alpha$.

Теорема 2.6 ([17], [2]). Значение произведения $\left\langle a_{1}, \ldots, a_{n}\right\rangle$ зависит только от классов когомологий элементов $a_{1}, \ldots, a_{n}$.

Определение 2.7 [17]. $n$-местное произведение Масси $\left\langle\alpha_{1}, \ldots, \alpha_{n}\right\rangle$ классов когомологий $\alpha_{1}, \ldots, \alpha_{n}$ определено, если определено произведение $\left\langle a_{1}, \ldots, a_{n}\right\rangle$ их представителей. В этом случае $\left\langle\alpha_{1}, \ldots, \alpha_{n}\right\rangle:=\left\langle a_{1}, \ldots, a_{n}\right\rangle$ как подмножества в $H^{*}(\mathscr{A})$.

Пример 2.8 (тройные произведения Масси). Пусть $\alpha, \beta$ и $\gamma-$ классы когомологий замкнутых элементов $a \in \mathscr{A}^{p}, b \in \mathscr{A}^{q}$ и $c \in \mathscr{A}^{r}$. Уравнение МаурераКартана для матрицы

$$
A=\left(\begin{array}{llll}
0 & 0 & 0 & 0 \\
c & 0 & 0 & 0 \\
g & b & 0 & 0 \\
h & f & a & 0
\end{array}\right)
$$

эквивалентно двум следующим уравнениям:

$$
d f=(-1)^{p+1} a \wedge b, \quad d g=(-1)^{q+1} b \wedge c .
$$

Следовательно, тройное произведение Масси $\langle\alpha, \beta, \gamma\rangle$ определено тогда и только тогда, когда

$$
\alpha \cdot \beta=\beta \cdot \gamma=0
$$

в когомологиях $H^{*}(\mathscr{A})$. Если эти условия выполнены, то произведение Масси $\langle\alpha, \beta, \gamma\rangle$ определено как подмножество в $H^{p+q+r-1}(\mathscr{A})$ следующего вида:

$$
\langle\alpha, \beta, \gamma\rangle=\left\{\left[(-1)^{p+1} a \wedge g+(-1)^{p+q} f \wedge c\right]\right\} .
$$

Матричные элементы $f$ и $g$ определяются формулами (5) с точностью до замкнутых элементов из $\mathscr{A}^{p+q-1}$ и $\mathscr{A}^{q+r-1}$ соответственно. Значит, значение тройного произведения Масси $\langle\alpha, \beta, \gamma\rangle$ представляет собой аффинное подпространство в $H^{p+q+r-1}(\mathscr{A})$, параллельное подпространству $\alpha \cdot H^{q+r-1}(\mathscr{A})+$ $H^{p+q-1}(\mathscr{A}) \cdot \gamma$.

Определение 2.9. Пусть $n$-местное произведение Масси $\left\langle\alpha_{1}, \ldots, \alpha_{n}\right\rangle$ определено. Оно называется тривиальным, если содержит нулевой класс когомологий: $0 \in\left\langle\alpha_{1}, \ldots, \alpha_{n}\right\rangle$.

Предложение 2.10 ([2], [17]). Пусть произведение Масси $\left\langle\alpha_{1}, \ldots, \alpha_{n}\right\rangle$ определено. Тогда все произведения $\left\langle\alpha_{l}, \ldots, \alpha_{q}\right\rangle, 1 \leqslant l<q \leqslant n, q-l<n-1$, определены и являются тривиальными.

Обозначим через $G L T_{n}(\mathbb{K})$ группу невырожденных нижнетреугольных $(n+1)$ $\times(n+1)$-матриц. 
Предложение 2.11. Пусть $A \in L T_{n}(\mathscr{A})$ - матрица формальной связности и $C$ - произвольная матрица из $G L T_{n}(\mathbb{K})$. Тогда матрица $C^{-1} A C \in$ $L T_{n}(\mathscr{A})$ удовлетворяет уравнению Маурера-Картана, т.е. она так же, как и $A$, является матрицей формальной связности. $I_{n}(\mathscr{A})$.

Доказательство. $d\left(C^{-1} A C\right)-\bar{C}^{-1} \bar{A} \bar{C} \wedge C^{-1} A C=C^{-1}(d A-\bar{A} \wedge A) C \in$

Определение 2.12. Две матрицы $A$ и $A^{\prime}$ формальных связностей из $L T_{n}(\mathscr{A})$ эквивалентны, если существует матрица $C \in G L(n+1, \mathbb{K})$, такая, что $A^{\prime}=$ $C^{-1} A C$.

Предложение 2.13. Пусть произведение Масси $\left\langle\alpha_{1}, \ldots, \alpha_{n}\right\rangle$ определено и $x_{1}, \ldots, x_{n}$ - набор из $n$ ненулевых констант. Тогда определено произведение Масси $\left\langle x_{1} \alpha_{1}, \ldots, x_{n} \alpha_{n}\right\rangle$; более того, справедливо следующее соотношение:

$$
\left\langle x_{1} \alpha_{1}, \ldots, x_{n} \alpha_{n}\right\rangle=x_{1} \cdots x_{n}\left\langle\alpha_{1}, \ldots, \alpha_{n}\right\rangle .
$$

Доказательство. Рассмотрим диагональную матрицу $C$, где по главной диагонали стоят сверху вниз последовательные произведения $x_{1} \cdots x_{n}, \ldots, x_{1} x_{2}$, $x_{1}, 1$. Взаимно однозначное отображение $A \rightarrow C^{-1} A C$ переводит произвольную матрицу формальной связности для произведения $\left\langle\alpha_{1}, \ldots, \alpha_{n}\right\rangle$ в некоторую матрицу связности, соответствующую уже произведению $\left\langle x_{1} \alpha_{1}, \ldots, x_{n} \alpha_{n}\right\rangle$. Очевидно, что $\tau\left(C^{-1} A C\right)=x_{1} \cdots x_{n} \cdot \tau(A)$.

Пусть $L T_{n}(\mathbb{K})$ - алгебра Ли строго нижнетреугольных $(n+1) \times(n+1)$-матриц над полем $\mathbb{K}$ нулевой характеристики, а $\mathfrak{g}$ - некоторая алгебра Ли.

Предложение 2.14. Матрица $А$ с элементами из $\mathfrak{g}^{*}$ определяет представление $\rho: \mathfrak{g} \rightarrow L T_{n}(\mathbb{K})$ тогда и только тогда, когда она удовлетворлет сильному уравнению Маурера-Картана $d A-\bar{A} \wedge A=0$.

Доказательство. Имеем $(d A-\bar{A} \wedge A)(x, y)=A([x, y])-[A(x), A(y)]$ для любых $x, y \in \mathfrak{g}$.

Рассмотрим произведение Масси вида

$$
\left\langle\omega_{1}, \ldots, \omega_{n}, \Omega\right\rangle, \quad \omega_{i} \in H^{1}(\mathfrak{g}), i=1, \ldots, n, \Omega \in H^{p}(\mathfrak{g}) .
$$

Если оно определено, то $n$-местное произведение $\left\langle\omega_{1}, \ldots, \omega_{n}\right\rangle$ тривиально. Это означает, что коцикл $\tau(A)$ для некоторой матрицы формальной связности $A$ является кограницей, и, значит, мы можем изменить элемент $a_{n+1, n}$ этой матрицы формальной связности так, что она будет удовлетворять сильному уравнению Маурера-Картана. Таким образом, существует гомоморфизм $\varphi: \mathfrak{g} \rightarrow L T_{n}(\mathbb{K})$. Это, в свою очередь, эквивалентно тому, что имеется $(n+1)$-мерный $\mathfrak{g}$-модуль $V$ с базисом $f_{1}, \ldots, f_{n+1}$, таким, что

$$
\mathfrak{g} f_{j} \in\left\langle f_{j+1}, \ldots, f_{n+1}\right\rangle, \quad j=1, \ldots, n, \quad \mathfrak{g} f_{n+1}=0 .
$$

Можно определить убывающую фильтрацию $\mathscr{F}$ комплекса $\left(C^{*}(\mathfrak{g}, V), d\right)$ :

$$
\mathscr{F}^{0} C^{*}(\mathfrak{g}, V) \supset \cdots \supset \mathscr{F}^{q} C^{*}(\mathfrak{g}, V) \supset \mathscr{F}^{q+1} C^{*}(\mathfrak{g}, V) \supset \ldots,
$$

где подпространство $\mathscr{F}^{q} C^{p+q}(\mathfrak{g}, V)$ определено как линейное подпространство $(p+q)$-форм $c$ в $C^{p+q}(\mathfrak{g}, V)$, таких, что $\operatorname{Im} c \subset\left\langle f_{q}, \ldots, f_{n+1}\right\rangle$. Фильтрация $\mathscr{F}$ совместима с $d$. Рассмотрим соответствующую спектральную последовательность $E_{r}^{p, q}$ (сходящуюся к когомологиям $H^{*}(\mathfrak{g}, V)$ ), для которой стандартным образом (см., например, [14]) доказывается следующее 
Предложение 2.15. $E_{1}^{p, q}=\left\langle f_{q}\right\rangle \otimes H^{p+q}(\mathfrak{g})$.

В дальнейшем мы будем называть эту спектральную последовательность $E_{r}^{p, q}$ спектральной последовательностью $(*)$.

Лемма 2.16. Пусть $\mathfrak{g}$ - алгебра Ли и определено $(n+1)$-местное произведение Масси

$$
\left\langle\omega_{1}, \ldots, \omega_{n}, \Omega\right\rangle, \quad \omega_{i} \in H^{1}(\mathfrak{g}), i=1, \ldots, n, \Omega \in H^{p}(\mathfrak{g}) .
$$

Тогда существует $(n+1)$-мерный $\mathfrak{g}$-модуль $V$ с выделенным базисом $f_{1}, \ldots$, $f_{n+1}$, удовлетворяющим условию (6), и спектральная последовательность (*), отвечающая фильтрачии (7) и сходящаяся к когомологиям $H^{*}(\mathfrak{g}, V)$, такая, что выполняются следующие условия:

$f_{1} \otimes \Omega \in E_{1}^{p-1,1}, \quad d_{1}\left(f_{1} \otimes \Omega\right)=\cdots=d_{n-1}\left(f_{1} \otimes \Omega\right)=0, \quad d_{n}\left(f_{1} \otimes \Omega\right)=f_{n+1} \otimes[c(A)]$,

где $d_{i}: E_{i}^{p, q} \rightarrow E_{i}^{p-i+1, q+i}$ есть $i$-й дифференциал спектральной последовательности $E_{r}^{p, q}$ и с $(A)$ является ассочиированным кочиклом матрицы формальной связности произведения $\left\langle\omega_{1}, \ldots, \omega_{n}, \Omega\right\rangle$.

Доказательство. Обозначим элементы матрицы $A$ следующим образом:

$$
A=\left(\begin{array}{ccccccc}
0 & 0 & 0 & \ldots & 0 & 0 & 0 \\
\Omega & 0 & 0 & \ldots & 0 & 0 & 0 \\
\Omega_{1} & \omega_{n} & 0 & \ldots & 0 & 0 & 0 \\
\Omega_{2} & a(n-1, n) & \omega_{n-1} & \ldots & 0 & 0 & 0 \\
\vdots & \vdots & \vdots & \ddots & \vdots & \vdots & \vdots \\
\Omega_{n-1} & a(2, n) & a(2, n-1) & \ldots & \omega_{2} & 0 & 0 \\
\Omega_{n} & a(1, n) & a(1, n-1) & \ldots & a(1,2) & \omega_{1} & 0
\end{array}\right) .
$$

Заметим, что для удобства вычислений нумерация элементов матрицы формальной связности $A$, которой пользовались перед этим, изменена. Уравнение Маурера-Картана дает нам следующую систему соотношений на элементы $\Omega_{i}$, стоящие в первом столбце матрицы $A$ :

$$
\begin{gathered}
d \Omega_{1}=\omega_{n} \wedge \Omega, \quad d \Omega_{2}=\omega_{n-1} \wedge \Omega_{1}+a(n-1, n) \wedge \Omega, \quad \ldots, \\
d \Omega_{n-1}=\omega_{2} \wedge \Omega_{n-1}+\cdots+a(2, n-2) \wedge \Omega_{2}+a(2, n-1) \wedge \Omega_{1}+a(2, n) \wedge \Omega .
\end{gathered}
$$

Мы напоминаем, что $\Omega_{n}$ может быть произвольной формой. При этом

$$
c(A)=\sum_{i=0}^{n-1} a(1, n-i) \wedge \Omega_{i}, \quad \Omega_{0}=\Omega, a(1,1)=\omega_{1} .
$$

С другой стороны, в коцепном комплексе $\left(C^{*}(\mathfrak{g}, V), \mathbf{d}\right)$ подпространство $C^{0}(\mathfrak{g}, V)$ совпадает с самим модулем $V$ и значение $\mathbf{d} f \in \operatorname{Hom}(\mathfrak{g}, V)=C^{1}(\mathfrak{g}, V)$ для произвольного $f \in V$ определяется формулой $\mathbf{d} f(X)=[X, f], X \in \mathfrak{g}$. Формула (1) для $v \in V$ и $\Omega \in \Lambda^{*}(\mathfrak{g})$ может быть переписана следующим образом:

$$
\mathbf{d}(v \otimes \Omega)=\mathbf{d} v \wedge \Omega-v \otimes d \Omega,
$$

где $d$ обозначает дифференциал комплекса $C^{*}(\mathfrak{g})$ с тривиальными коэффициентами. Следовательно, $\mathbf{d} f_{n+1}=0$ и

$\mathbf{d} f_{i}=f_{i+1} \otimes \omega_{n-i+1}+f_{i+2} \otimes a(n-i, n-i+1)+\cdots+f_{n+1} \otimes a(1, n-i+1), \quad i=1, \ldots, n$. 
Отсюда вытекает, что $\mathbf{d}\left(f_{1} \otimes \Omega\right)=f_{2} \otimes \omega_{n} \wedge \Omega+\ldots$, где многоточие стоит вместо членов более высокой фильтрации. Окончательно получаем искомую формулу:

$$
\mathbf{d}\left(\sum_{i=1}^{n} f_{i} \otimes \Omega_{i-1}\right)=f_{n+1} \otimes\left(\sum_{i=0}^{n-1} a(1, n-i) \wedge \Omega_{i}\right)=f_{n+1} \otimes c(A) .
$$

Верно и утверждение, обратное к предыдущей лемме.

Лемма 2.17. Пусть $\mathfrak{g}$ - алгебра Ли и существует $(n+1)$-мерный $\mathfrak{g}$-модуль $V$ с выделенным базисом $f_{1}, \ldots, f_{n+1}$, удовлетворяющим условию (6). Предположим при этом, что дифберенииалы $d_{1}, \ldots, d_{n-1}$ спектральной последовательности (*) обращаются в нуль на элементе $f_{1} \otimes \Omega$. Тогда существуют такие классы $\omega_{1}, \ldots, \omega_{n}$ одномерных когомологий из $H^{1}(\mathfrak{g})$, что определено $(n+1)$ местное произведение Масси $\left\langle\omega_{1}, \ldots, \omega_{n}, \Omega\right\rangle$, причем $f_{n+1} \otimes[c(A)]=d_{n}\left(f_{1} \otimes \Omega\right)$.

Доказательство полностью повторяет, только в обратном направлении, доказательство леммы 2.16.

Теорема 2.18 [13]. Пусть $g_{ \pm}^{k}$ - нетривиальный коцикл в $H_{\left(3 k^{2} \pm k\right) / 2}^{k}\left(L_{1}\right)$. Для любого $k \geqslant 2$ имеют место следующие включения:

$$
g_{-}^{k} \in\langle\underbrace{e^{1}, \ldots, e^{1}}_{2 k-1}, g_{+}^{k-1},\rangle, \quad g_{+}^{k} \in\langle\underbrace{e^{1}, \ldots, e^{1}}_{3 k-1}, g_{+}^{k-1}\rangle .
$$

Замечание. Используя результаты самой работы [13], несложно установить, что все произведения Масси из теоремы 2.18 являются тривиальными.

Следующая попытка приблизиться к доказательству гипотезы Бухштабера была предпринята в 2000 г.: И. В. Артельных анонсировал следующую теорему.

Теорема 2.19 [1]. Имеются нетривиальные произведения Масси

$$
g_{-}^{k} \in\langle\underbrace{e^{2}, \ldots, e^{2}}_{k-1}, g_{+}^{k-1}, e^{1}\rangle, \quad k \geqslant 2, \quad g_{+}^{2 l+1} \in\langle\underbrace{e^{2}, \ldots, e^{2}}_{3 l+1}, g_{+}^{2 l}\rangle, \quad l \geqslant 1 .
$$

Видно, что Артельных не удалось найти нетривиальные произведения Масси для четномерных классов $g_{+}^{2 l}$. Кроме того, его работа [1] - краткая заметка, не содержащая полных доказательств, которые, к сожалению, не были опубликованы в дальнейшем.

\section{§3. Основная теорема}

Теорема 3.1. Когомологии $H^{*}\left(L_{1}\right)$ рекуррентно порождаются двумя базисными элементами $e^{1}, e^{2}$ одномерных когомологий $H^{1}\left(L_{1}\right)$ с помощью двух серий нетривиальных произведений Масси. Более точно:

1) Тройное произведение Масси $\left\langle e^{1}, e^{2}, e^{2}\right\rangle$ однозначно определено и определяет нетривиальный класс когомологий $g_{-}^{2}=\left\langle e^{1}, e^{2}, e^{2}\right\rangle \in H_{5}^{2}\left(L_{1}\right)$.

2) Пятиместное произведение $\left\langle e^{1}, e^{2}, e^{1}, e^{1}, e^{2}\right\rangle$ является нетривиальным u представляет собой аффинную прямую $\left\{g_{+}^{2}+t g_{-}^{2}, t \in \mathbb{K}\right\}$ на двумерной плоскости $H^{2}\left(L_{1}\right)$, где $g_{+}^{2}$ обозначает некоторый нетривиальный элемент из $H_{7}^{2}\left(L_{1}\right)$. Возвмем произвольный элемент $\tilde{g}_{+}^{2} \in\left\langle e^{1}, e^{2}, e^{1}, e^{1}, e^{2}\right\rangle$ в качестве второго базисного вектора в $H^{2}\left(L_{1}\right)$. 
3) Предположим, что уже построен некоторый базис $g_{-}^{k}, \tilde{g}_{+}^{k}$ пространства $H^{k}\left(L_{1}\right), k \geqslant 2$, такой, что класс когомологий $g_{-}^{k}$ порождает одномерное подпространство $H_{\left(3 k^{2}-k\right) / 2}^{k}\left(L_{1}\right)$. Тогда $(2 k+1)$-местное произведение Масси

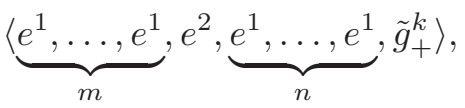

где $m, n$ - произвольные натуральные числа, такие, что $m+n=2 k-1$, однозначно определено и дает образующую $g_{-}^{k+1}$ одномерного подпространства $H_{\left(3(k+1)^{2}-(k+1)\right) / 3}^{k+1}\left(L_{1}\right)$.

$(3 k+2)$-местное произведение Масси

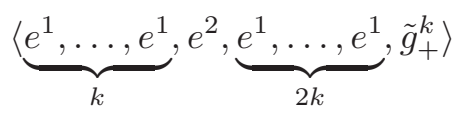

является нетривиальным и представляет собой афбинную прямую на двумерной плоскости $H^{k+1}\left(L_{1}\right)$, параллельную одномерному подпространству $H_{\left(3(k+1)^{2}-(k+1)\right) / 2}^{k+1}\left(L_{1}\right)$. Возвмем в $\langle\underbrace{e^{1}, \ldots, e^{1}}_{k}, e^{2}, \underbrace{e^{1}, \ldots, e^{1}}_{2 k}, \tilde{g}_{+}^{k}\rangle$ произвольный элемент $\tilde{g}_{+}^{k+1} ;$ тогда $g_{-}^{k+1}$ и $\tilde{g}_{+}^{k+1}$ образуют базис в $H^{k+1}\left(L_{1}\right)$.

Доказательство. 1) Для любой матрицы формальной связности $A^{\prime}$, соответствующей тройному произведению Масси $\left\langle e^{1}, e^{2}, e^{2}\right\rangle$, которое определено, см. пример 2.8, ассоциированный коцикл $c\left(A^{\prime}\right)$ будет равен $-e^{2} \wedge e^{3}+\alpha d e^{3}$, где $\alpha$ - некоторая константа, зависящая от матрицы $A^{\prime}$. Таким образом, $\left\langle e^{1}, e^{2}, e^{2}\right\rangle=$ $-\left[e^{2} \wedge e^{3}\right] \neq 0$ в $H_{5}^{2}\left(L_{1}\right)$ и, значит, $\left\langle e^{1}, e^{2}, e^{2}\right\rangle$ однозначно определено.

2) Рассмотрим следующую матрицу формальной связности $A$ :

$$
A=\left(\begin{array}{cccccc}
0 & 0 & 0 & 0 & 0 & 0 \\
-e^{2} & 0 & 0 & 0 & 0 & 0 \\
2 e^{3} & -2 e^{1} & 0 & 0 & 0 & 0 \\
-e^{4}-t e^{2} & -e^{2} & -e^{1} & 0 & 0 & 0 \\
0 & e^{4} & e^{3} & e^{2} & 0 & 0 \\
0 & e^{5} & e^{4} & e^{3} & e^{1} & 0
\end{array}\right) .
$$

Она отвечает произведению $\left\langle e^{1}, e^{2},-e^{1},-2 e^{1},-e^{2}\right\rangle=-2\left\langle e^{1}, e^{2}, e^{1}, e^{1}, e^{2}\right\rangle$, a eе ассоциированный коцикл $c(A)$ равен

$$
c(A)=\left(e^{2} \wedge e^{5}-3 e^{3} \wedge e^{4}\right)+t e^{2} \wedge e^{3} \in g_{+}^{2}+t g_{-}^{2},
$$

где значение параметра $t$ может быть произвольным. С другой стороны, для произвольной матрицы формальной связности $A^{\prime}$, отвечающей тому же самому произведению Масси, ассоциированный коцикл $c\left(A^{\prime}\right)$ будет всегда иметь вид $c\left(A^{\prime}\right)=\left(e^{2} \wedge e^{5}-3 e^{3} \wedge e^{4}\right)+\ldots$, где вместо слагаемых с весом меньше семи мы поставили многоточие. Эти слагаемые как раз и отвечают за неоднозначность произведения $\left\langle e^{1}, e^{2}, e^{1}, e^{1}, e^{2}\right\rangle$, которая может быть лишь вида $t g_{-}^{2}$ и уже включена в формулу (12).

Индуктивный переход. Предположим, что мы уже построили некоторый базис $g_{-}^{k}, \tilde{g}_{+}^{k}$ пространства $H^{k}\left(L_{1}\right), k \geqslant 2$, такой, что класс когомологий $g_{-}^{k}$ порож- 
дает одномерное подпространство $H_{\left(3 k^{2}-k\right) / 2}^{k}\left(L_{1}\right)$. Покажем сначала, что произведение Масси $\langle\underbrace{e^{1}, \ldots, e^{1}}_{m}, e^{2}, \underbrace{e^{1}, \ldots, e^{1}}_{n}\rangle$ определено и тривиально. Построение для него матрицы формальной связности $A$ равносильно нахождению $L_{1}$-модуля $V^{m, n}$ с выделенным базисом $f_{-m-1}, \ldots, f_{-1}, f_{1}, \ldots, f_{n}$ ( $f_{0}$ отсутствует), в котором матрицы всех операторов $e_{i}$ имеют строго нижнетреугольный вид, причем для $-m-1 \leqslant j \leqslant n-1, j \neq-1,0$, и $-m-1 \leqslant i \leqslant n-2, i \neq 0$,

$$
e_{1} f_{j}=f_{j+1}+\alpha_{j}^{j+2} f_{j+2}+\cdots+\alpha_{j}^{n} f_{n}, \quad e_{2} f_{i}=b_{i} f_{i+2}+\beta_{i}^{i+3} f_{i+3}+\cdots+\beta_{i}^{n} f_{n}
$$

а также $b_{-1}=1$ и $e_{1} f_{-1}=\alpha_{-1}^{2} f_{2}+\cdots+\alpha_{-1}^{n} f_{n}$. Удобно рассмотреть прямую сумму $\widetilde{V}^{m, n}=V^{m, n} \oplus\left\langle f_{0}\right\rangle$ модуля $V^{m, n}$ и одномерного тривиального модуля $\left\langle f_{0}\right\rangle$. Теперь флаг подпространств $\widetilde{V}_{i}^{m, n}=\left\langle f_{i}, \ldots, f_{n}\right\rangle, i=-m-1, \ldots, n$, определяет убывающую фильтрацию модуля $\widetilde{V}^{m, n}: e_{i} \widetilde{V}_{j}^{m, n} \subset \widetilde{V}_{i+j}^{m, n}$, и относительно нее можно рассмотреть ассоциированный градуированный модуль $\widetilde{V}_{\mathrm{gr}}^{m, n}$ (для базиса которого сохраним старые обозначения), причем градуированный $L_{1}$-модуль $\widetilde{V}_{\mathrm{gr}}^{m, n}$ по-прежнему будет содержать $\left\langle f_{0}\right\rangle$ в качестве прямого слагаемого (как $L_{1}$-модуля):

$$
\widetilde{V}_{\mathrm{gr}}^{m, n}=V_{\mathrm{gr}}^{m, n} \oplus\left\langle f_{0}\right\rangle, \quad \widetilde{V}_{\mathrm{gr}}^{m, n}=\bigoplus_{i=-m-1}^{n}\left(\widetilde{V}_{\mathrm{gr}}^{m, n}\right)_{i}, \quad\left(\widetilde{V}_{\mathrm{gr}}^{m, n}\right)_{i}=\widetilde{V}_{i}^{m, n} / \widetilde{V}_{i+1}^{m, n}=\left\langle f_{i}\right\rangle .
$$

Модуль $\widetilde{V}_{\mathrm{gr}}^{m, n}$ также задается формулами $(13)$, в которых надо положить $\alpha_{j}^{l}=$ $\beta_{i}^{s}=b_{0}=0$. Оказывается, что градуированный модуль $\widetilde{V}_{\mathrm{gr}}^{m, n}$ определен однозначно с точностью до изоморфизма. Предъявим его сразу. Для этого определим сначала бесконечномерный градуированный $L_{1}$-модуль $\widetilde{V}_{\mathrm{gr}}$ при помощи базиса $\left\{f_{j}, j \in \mathbb{Z}\right\}$ и соотношений

$$
e_{i} f_{j}= \begin{cases}j f_{i+j}, & j \geqslant 0, \\ (i+j) f_{i+j}, & i+j \leqslant 0, j<0, \\ f_{i+j}, & i+j>0, j<0 .\end{cases}
$$

Хотя сам модуль $\widetilde{V}_{\text {gr }}$ бесконечномерный, его конечномерный подфактор

$$
\tilde{V}_{\mathrm{gr}}^{m, n}=\left(\bigoplus_{i \geqslant-m-1}\left\langle f_{i}\right\rangle\right) /\left(\bigoplus_{i \geqslant n}\left\langle f_{i}\right\rangle\right)
$$

как раз и обладает нужными нам свойствами. Докажем теперь его единственность.

Лемма 3.2. Пусть $\widetilde{V}^{m, n}=\bigoplus_{i=-m-1}^{n} V_{i}^{m, n}-$ градуированный конечномерный $L_{1}$-модуль с одномерными однородными компонентами $\widetilde{V}_{i}^{m, n}$ с $m \geqslant 2 u$ $n \geqslant 5$, такой, что

$$
\begin{gathered}
e_{1} \widetilde{V}_{i}^{m, n}=\widetilde{V}_{i+1}^{m, n}, \quad i \neq-1,0, n, \quad e_{1} \widetilde{V}_{-1}^{m, n}=e_{1} \widetilde{V}_{0}^{m, n}=e_{1} \widetilde{V}_{n}^{m, n}=0, \\
\tilde{e}_{2} V_{j}^{m, n} \subset V_{j+2}^{m, n}, \quad j \neq-2,-1, n, n-1, \\
e_{2} \widetilde{V}_{-1}^{m, n}=\widetilde{V}_{1}^{m, n}, \quad e_{2} \widetilde{V}_{-2}^{m, n}=e_{2} \widetilde{V}_{n-1}^{m, n}=e_{2} \widetilde{V}_{n}^{m, n}=0 .
\end{gathered}
$$


Тогда градуированный модуль $\widetilde{V}^{m, n}$ изоморфен подфактору $\widetilde{V}_{\mathrm{gr}}^{m, n}$.

Доказательство. Рассмотрим новый базис $\tilde{e}_{i}$ алгебры $L_{1}$ :

$$
\tilde{e}_{1}=e_{1}, \quad \tilde{e}_{2}=6 e_{2}, \quad \tilde{e}_{i}=6(i-2) ! e_{i}, \quad i \geqslant 3 .
$$

Векторы нового базиса связаны между собой следующими рекуррентными соотношениями:

$$
\left[\tilde{e}_{1}, \tilde{e}_{i}\right]=\tilde{e}_{i+1}, \quad i \geqslant 2 .
$$

Аналогичным образом выбираем специальный базис $f_{-m-1}, \ldots, f_{-1}, f_{0}, f_{1}$, $\ldots, f_{n}$ в модуле $V^{m, n}$ со следующими свойствами:

$$
\begin{gathered}
\tilde{e}_{1} f_{i}=f_{i+1}, \quad i \neq-1,0, n, \quad \tilde{e}_{1} f_{-1}=\tilde{e}_{1} f_{0}=\tilde{e}_{1} f_{n}=0, \\
\tilde{e}_{2} f_{i}=b_{i} f_{i+2}, \quad i \neq n, n-1, \quad b_{-2}=0, \quad b_{-1}=b_{0}=1 .
\end{gathered}
$$

Мы смогли нормировать константы $b_{-1}=b_{0}=1$, так как векторы $f_{1}$ и $f_{0}$ могут быть выбраны произвольно.

Известно, что алгебра $L_{1}$ порождается двумя элементами $\tilde{e}_{1}, \tilde{e}_{2}$ со следующими определяющими соотношениями (тут трудно выделить самую первую ссылку на это почти очевидное утверждение, см., например, [4]):

$$
\left[\tilde{e}_{2}, \tilde{e}_{3}\right]=\tilde{e}_{5}, \quad\left[\tilde{e}_{2}, \tilde{e}_{5}\right]=\frac{9}{10} \tilde{e}_{7},
$$

где элементы $\tilde{e}_{3}, \tilde{e}_{5}, \tilde{e}_{7}$ определены при помощи соотношений (15).

Таким образом, всякий градуированный $L_{1}$-модуль $V^{m, n}$ определяется набором констант $b_{-m-1}, \ldots, b_{n-2}$ и следующей системой уравнений (см. также работу [4]):

$$
\begin{array}{ll}
R_{i}^{5}: & \left(\left[\tilde{e}_{2}, \tilde{e}_{3}\right]-\tilde{e}_{5}\right) f_{i}=0, \quad i=-m-1, \ldots, n-5, \\
R_{j}^{7}: & \left(\left[\tilde{e}_{2}, \tilde{e}_{5}\right]-\frac{9}{10} \tilde{e}_{7}\right) f_{j}=0, \quad j=-m-1, \ldots, n-7 .
\end{array}
$$

Напомним, что по условию $m \geqslant 2$ и $n \geqslant 5$. Легко видеть, что четыре уравнения $R_{-2}^{5}, R_{-1}^{5}, R_{0}^{5}, R_{-2}^{7}$ запишутся следующим образом:

$$
\begin{gathered}
-b_{-1} b_{1}-b_{-2} b_{0}+3 b_{-1}=0, \quad 2 b_{-1} b_{2}-b_{-1} b_{1}-b_{-1}=0, \\
2 b_{0} b_{3}-b_{0} b_{2}-b_{0}=0, \quad-3 b_{-1} b_{3}-b_{-2} b_{0}+\frac{9}{2} b_{-1}=0 .
\end{gathered}
$$

Система (17) имеет единственное решение $b_{1}=3, b_{2}=2, b_{3}=3 / 2$. Относительно $b_{-3}$ уравнение $R_{-3}^{5}: b_{-2} b_{0}-b_{-3} b_{-1}-3 b_{-1}=0$ имеет единственное решение $b_{-3}=-3$. У уравнения $R_{-4}^{5}$ также лишь одно решение $b_{-4}=-2$.

Наконец определим $b_{-5}$. Уравнение $R_{-5}^{5}: 2 b_{-5} b_{-2}-b_{-4} b_{-2}+b_{-2}=0$ вырождается, а второе уравнение, $R_{-5}^{7}:-b_{0} b_{-2}-3 b_{-5} b_{-1}-\frac{9}{2} b_{-1}=0$, имеет единственное решение $b_{-5}=-3 / 2$.

Продолжая этот процесс рекуррентно, мы находим все коэффициенты $b_{-m-1}$, $\ldots, b_{n-2}$, отвечающие подфактору $\widetilde{V}^{m, n}$. Представим их в виде таблицы:

\begin{tabular}{|c|c|c|c|c|c|c|c|c|c|c|}
\hline$b_{-m-1}$ & $\ldots$ & $b_{-4}$ & $b_{-3}$ & $b_{-2}$ & $b_{-1}$ & $b_{0}$ & $b_{1}$ & $b_{2}$ & $\ldots$ & $b_{n-2}$ \\
\hline$-6 / m$ & $\ldots$ & -2 & -3 & 0 & 1 & 1 & 3 & 2 & $\ldots$ & $6 /(n-1)$ \\
\hline
\end{tabular}

При $i<-5$ или $i>0$ уравнение $R_{i}^{5}$ будет иметь единый вид:

$$
R_{i}^{5}: \quad b_{i+3}\left(b_{i}-b_{i+1}\right)-b_{i}\left(b_{i+2}-b_{i+3}\right)=b_{i}-3 b_{i+1}+3 b_{i+2}-b_{i+3} .
$$


Коэффициент при $b_{i}$ равен $1+b_{i+2}-2 b_{i+3}=\frac{i(i+1)}{(i+3)(i+4)}$, если предположить по индукции, что $b_{i+2}=\frac{6}{i+3}, b_{i+3}=\frac{6}{i+4}$. Следовательно, значения $b_{i}$ при $i<5$ будут определяться однозначно с помощью $R_{i}^{5}$ и будут равны $b_{i}=\frac{6}{i+1}$. Аналогичным образом разбирается случай $i>0$ (уравнение $R_{i}^{5}$ относительно неизвестной $\left.b_{i+3}\right)$.

Следствие 3.3. Существует такая выделенная матрица формальной связности $A^{\mathrm{gr}}$, отвечающая тривиальному произведению Масси $\underbrace{e^{1}, \ldots, e^{1}}_{m}, e^{2}$, $\underbrace{e^{1}, \ldots, e^{1}}\rangle, m \geqslant 2, n \geqslant 5$, что для произвольного элемента $a_{i, j}^{\prime}, i>j$, любой другой матрицъ формальной связности $A^{\prime}$, соответствующей тому же самому произведению Масси, выполняется равенство

$$
a_{i, j}^{\prime}=a_{i, j}^{\mathrm{gr}}+\delta(i, j),
$$

где $\delta(i, j)$ обозначает линейную комбинацию базисных функиионалов $e^{r}$, индексы $r$ которых строго менъше веса элемента $a_{i, j}^{\mathrm{gr}}$ матрицы формалъной связности $A^{\mathrm{gr}}$.

Замечание. Нам нужен собственно модуль $V_{\mathrm{gr}}^{m, n}$ для построения произведений Масси, но при этом удобнее вычислять когомологии $H^{*}\left(L_{1}, \tilde{V}_{\mathrm{gr}}^{m, n}\right)$. А связь между $H^{*}\left(L_{1}, \widetilde{V}_{\mathrm{gr}}^{m, n}\right)$ и $H^{*}\left(L_{1}, V_{\mathrm{gr}}^{m, n}\right)$ очевидна:

$$
H^{*}\left(L_{1}, \widetilde{V}_{\mathrm{gr}}^{m, n}\right)=H^{*}\left(L_{1}, V_{\mathrm{gr}}^{m, n}\right) \oplus\left(\left\langle f_{0}\right\rangle \otimes H^{*}\left(L_{1}\right)\right) .
$$

3) Рассмотрим теперь модуль $\tilde{V}_{\mathrm{gr}}^{m, n}=\left\langle f_{-m-1}, \ldots, f_{n}\right\rangle$ при $m+n=2 k-1$. В компоненте веса $-m-1-\frac{3 k^{2}+k}{2}$ в спектральной последовательности $(*)$, отвечающей фильтрации (7) и сходящейся к когомологиям $H^{*}\left(L_{1}, \widetilde{V}_{\mathrm{gr}}^{m, n}\right)$, среди дифференциалов $d_{r}^{k+m+1,-m-1}: E_{r}^{k+m+1,-m-1} \rightarrow E_{r}^{-k+m-r+2,-m-1+r}, r=$ $1,2, \ldots$, есть только один, $d_{2 k+1}^{k+m+1,-m-1}$, который может оказаться нетривиальным:

$$
\left\langle g_{+}^{k} \otimes f_{-m-1}\right\rangle \stackrel{d_{2 k+1}}{\longrightarrow}\left\langle g_{-}^{k+1} \otimes f_{n}\right\rangle
$$

Действительно, в $E_{1}^{*, *}$ подпространство элементов веса $-m-1-\frac{3 k^{2}+k}{2}$ двумерно и совпадает с линейной оболочкой $\left\langle g_{+}^{k} \otimes f_{-m-1}, g_{-}^{k+1} \otimes f_{n}\right\rangle$. При этом, согласно лемме $2.17, d_{2 k+1}\left(g_{+}^{k} \otimes f_{-m-1}\right)=c\left(A^{\mathrm{gr}}\right) \otimes f_{n}$.

Лемма 3.4. Дифференциал $d_{2 k+1}$, а значит, и коцикл $c\left(A^{\mathrm{gr}}\right)$, является нетривиалъным. Следовательно, в силу однородности $c\left(A^{\mathrm{gr}}\right)=g_{-}^{k+1}$.

Мы отнесем доказательство этой леммы в последний параграф.

Предложение 3.5. Произведение Масси $\langle\underbrace{e^{1}, \ldots, e^{1}}_{m}, e^{2}, \underbrace{e^{1}, \ldots, e^{1}}_{n}, g_{+}^{k}+t g_{-}^{k}\rangle$, $m+n=2 k-1$, однозначно определено и равняется нетривиальному однородному элементу $g_{-}^{k+1}$ из $H_{\left(3(k+1)^{2}-(k+1)\right) / 2}^{k+1}\left(L_{1}\right)$ при любых значениях числового параметра $t$. 
Доказательство. Матрица $\tilde{A}$ произвольной формальной связности произведения $\left\langle e^{1}, \ldots, e^{1}, e^{2}, e^{1}, \ldots, e^{1}, g_{+}^{k}+t g_{-}^{k}\right\rangle$ получается добавлением к некоторой матрице связности $A$ произведения $\left\langle e^{1}, \ldots, e^{1}, e^{2}, e^{1}, \ldots, e^{1}\right\rangle$ верхней нулевой строки и левого столбца $\Omega$ так, как мы это делали в доказательстве леммы 2.16:

$$
\tilde{A}=\left(\begin{array}{ccccc}
0 & 0 & \ldots & 0 & 0 \\
\gamma_{+}^{k}+t \gamma_{-}^{k} & 0 & \ddots & 0 & 0 \\
\Omega_{1} & \omega_{n} & \ddots & \ddots & 0 \\
\vdots & \vdots & \ddots & 0 & \vdots \\
\Omega_{m+n+2} & a(1, m+n+2) & \ldots & \omega_{-m-1} & 0
\end{array}\right),
$$

где $\left(\omega_{-m-1}, \ldots, \omega_{n}\right)=\left(e_{1}, \ldots, e_{1}, e_{2}, e_{1}, \ldots, e_{1}\right)$, а коциклы $\gamma_{+}^{k}$ и $\gamma_{-}^{k}$ представляют классы когомологий $g_{+}^{k}$ и $g_{-}^{k}$ соответственно. $k$-формы $\Omega_{i}$ первого столбца находятся рекуррентно, начиная с уравнения $d \Omega_{1}=e^{1} \wedge\left(\gamma_{+}^{k}+t \gamma_{-}^{k}\right)$. Заметим, что $k$-форма $\Omega_{1}$ определена с точностью до замкнутого элемента $\alpha \gamma_{-}^{k}+\beta \gamma_{+}^{k}+d c$, добавка точной формы $d c$ не изменит класс когомологий $c(\tilde{A})$. С другой стороны, максимальный вес слагаемого в разложении $\Omega_{1}$ никак не может быть меньше $\frac{3 k^{2}+k}{2}+1$. Продолжая последовательно эту процедуру и учитывая следствие 3.3 , получаем, что максимальный вес слагаемого в разложении коцикла $c(\tilde{A})$ (по модулю точных форм) всегда равен $\frac{3 k^{2}+k}{2}+2 k-1=\frac{3(k+1)^{2}-(k+1)}{2}$, а значит, $[c(A)]=\left[c\left(A^{\mathrm{gr}}\right)\right]=g_{-}^{k+1}$, потому что все однородные слагаемые в разложении коцикла $c(\tilde{A})$ с весами меньше $\frac{3(k+1)^{2}-(k+1)}{2}$ являются точными $(k+1)$-формами.

4) Рассмотрим конечномерный градуированный $L_{1}$-модуль $\widetilde{V}^{2 k, k+1}$. В члене $E_{1}^{*, *}$ подпространство элементов веса $-\frac{3(k+1)^{2}-(k+1)}{2}$ двумерно и порождено $g_{+}^{k} \otimes f_{-2 k-1}$ и $g_{+}^{k+1} \otimes f_{k+1}$. Таким образом, в этой градуировке у спектральной последовательности $(*)$ среди дифференциалов

$$
d_{r}^{3 k+1,-2 k-1}: E_{r}^{3 k+1,-2 k-1} \rightarrow E_{r}^{3 k-r+2,-2 k-1+r}, \quad r=1,2, \ldots,
$$

есть только один потенциально ненулевой дифференциал

$$
d_{3 k+2}^{3 k+1,-2 k-1}: E_{3 k+2}^{3 k+1,-2 k-1} \rightarrow E_{3 k+2}^{0, k+1}
$$

(заметим, что дифференциал $d_{2 k+1}$ тоже тривиален в этом случае):

$$
\left\langle g_{+}^{k} \otimes f_{-2 k-1}\right\rangle \stackrel{d_{3 k+2}}{\longrightarrow}\left\langle g_{+}^{k+1} \otimes f_{k+1}\right\rangle, \quad d_{3 k+2}\left(g_{+}^{k} \otimes f_{-2 k-1}\right)=c\left(A^{\mathrm{gr}}\right) \otimes f_{k+1} .
$$

Лемма 3.6. Дифференииал $d_{3 k+2}\left(u\right.$, следовательно, коиикл $\left.\left[c\left(A^{\mathrm{gr}}\right)\right]\right)$ является нетривиальным.

Эта лемма также будет доказана отдельно в последнем параграфе.

Предложение 3.7. Произведение Масси $\langle\underbrace{e^{1}, \ldots, e^{1}}_{k}, e^{2}, \underbrace{e^{1}, \ldots, e^{1}}_{2 k}, g_{+}^{k}+t g_{-}^{k}\rangle$ нетривиально и представляет собой афбинную прямую $\left\{g_{+}^{k+1}+u g_{-}^{k+1}, u \in \mathbb{K}\right\}$ на двумерной плоскости $H^{k+1}\left(L_{1}\right)$ при любом значении параметра $t$. 
Доказательство. Сохраним обозначения из леммы 3.5. Мы без препятствий находим однородные формы $\Omega_{i}, i=1, \ldots, 2 k-2$, причем мы специально поставили в произведении Масси элемент $e^{2}$ перед $2 k$ экземпляров $e^{1}$, чтобы искать однородную форму $\Omega_{2 k-1}$ уже с весом $-\frac{1}{2}\left(3(k+1)^{2}-(k+1)\right)-1$, а все замкнутые формы с таким весом являются точными. Те же аргументы, что и в лемме 3.5 , показывают, что наше произведение Масси представляет собой некоторое подмножество аффинной прямой $\left\{g_{+}^{k+1}+u g_{-}^{k+1}, u \in \mathbb{K}\right\}$, причем можно показать, что реализуются все точки прямой.

\section{§4. Особые векторы модулей Верма и резольвента Фейгина-Фукса-Роча-Карриди-Уоллаха}

Модулем Верма над алгеброй Вирасоро называется Vir-модуль $V(h, c)$, свободный над универсальной обертывающей $U_{L_{1}}$ подалгебры $L_{1} \subset$ Vir и порожденный некоторым вектором $v$, таким, что

$$
z v=c v, \quad e_{0} v=h v, \quad e_{i} v=0, \quad i<0,
$$

где $c, h \in \mathbb{C}$. Как векторное пространство $V(h, c)$ может быть задан своим бесконечным базисом

$$
v, e_{i_{1}} \cdots e_{i_{s}} v, \quad i_{1} \geqslant \cdots \geqslant i_{s}, s \geqslant 1 .
$$

Модуль Верма $V(h, c)$ является $\mathbb{Z}_{+}$-градуированным модулем:

$$
V(h, c)=\bigoplus_{n=0}^{+\infty} V_{n}(h, c), \quad V_{n}(h, c)=\left\langle e_{i_{1}} \cdots e_{i_{s}} v, i_{1}+\cdots+i_{s}=n\right\rangle .
$$

$V_{n}(h, c)$ образует собственное подпространство оператора $e_{0}$, отвечающее собственному значению $(h+n)$,

$$
e_{0}\left(e_{i_{1}} \cdots e_{i_{s}} v\right)=\left(h+i_{1}+\cdots+i_{s}\right) e_{i_{1}} \cdots e_{i_{s}} v .
$$

Кроме этого, $z w=c w$ для любого $w \in V(h, c)$.

Определение 4.1. Ненулевой вектор $w \in V(h, c)$ называется особым, если $e_{i} w=0$ для всех $i<0$.

Однородный особый вектор $w \in V_{n}(h, c)$ порождает в $V(h, c)$ подмодуль, изоморфный $V(h+n, c)$.

Теорема 4.2 ([16], [11], [12]). В модуле Верма $V(h, c)$ тогда и толъко тогда существует особый вектор $w \in V_{n}(h, c)$ с градуировкой не выше $n$, когда найдутся два натуральных числа р и q, а также комплексное число $t$, такие, что

$$
\begin{gathered}
p q \leqslant n, \quad c=c(t)=13+6 t+6 t^{-1}, \\
h=h_{p, q}(t)=\frac{1-p^{2}}{4} t+\frac{1-p q}{2}+\frac{1-q^{2}}{4} t^{-1} .
\end{gathered}
$$

Верно, в частности, и такое утверждение [15]: при фиксированных натуральных числах $p$ и $q$ и произвольном комплексном $t$ модуль Верма $V\left(h_{p, q}(t), c(t)\right)$ содержит особый вектор $w_{p, q}(t)$ градуировки $p q$, причем этот вектор определен однозначно с точностью до умножения на скалярный множитель:

$$
w_{p, q}(t)=S_{p, q}(t) v=\sum_{|I|=p q} a_{I}^{p, q}(t) e_{I} v=\sum_{i_{1}+\cdots+i_{s}=p q} a_{i_{1}, \ldots, i_{s}}^{p, q}(t) e_{i_{1}} \cdots e_{i_{s}} v,
$$


где $S_{p, q}(t)$ обозначает некоторый элемент универсальной обертывающей алгебры $U\left(L_{1}\right)$. Коэффициенты $a_{I}^{p, q}(t)$ зависят полиномиально от $t$ и $t^{-1}$. Мы предполагаем, что коэффициент $a_{1, \ldots, 1}^{p, q}(t)$ равен единице. Очевидно, что $S_{p, q}(t)=$ $S_{q, p}\left(t^{-1}\right)$.

Предложение 4.3 ([16], [11], [12]). В однородном подпространстве $V_{n}(0,0)$ модуля Верма $V(0,0)$ тогда и толъко тогда существует особый вектор $w_{n}$, когда $n$ является пентагональным числом $n=e_{ \pm}(k)=\frac{3 k^{2} \pm k}{2}$.

Согласно теореме 4.2, чтобы у модуля Верма $V(h, 0)$ с $c=0$ был особый вектор $w_{p, q}(t)$, должно выполняться равенство $t=-3 / 2$ (или $t=-2 / 3$ ). Мы зафиксируем значение $t=-3 / 2$ и для удобства обозначений вместо $S_{p, q}(-3 / 2)$ будем писать $S_{p, q}$. Обозначим через $V\left(\frac{3 k^{2} \pm k}{2}\right)$ подмодуль в модуле Верма $V(0,0)$, порожденный особым вектором градуировки $\frac{3 k^{2} \pm k}{2}$. Подмодуль $V\left(\frac{3 k^{2} \pm k}{2}\right)$ изоморфен модулю Верма $V\left(\frac{3 k^{2} \pm k}{2}, 0\right)$.

Предложение 4.4 ([19], [11]). У системы подмодулей $V\left(\frac{3 k^{2} \pm k}{2}\right)$ имеются следующие важные свойства:

1) сумма $V(1)+V(2)$ является подпространством коразмерности один в $V(0,0)$;

2) $V\left(\frac{3 k^{2}-k}{2}\right) \cap V\left(\frac{3 k^{2}+k}{2}\right)=V\left(\frac{3(k+1)^{2}-(k+1)}{2}\right)+V\left(\frac{3(k+1)^{2}+(k+1)}{2}\right), k \geqslant 1$.

Можно непосредственно убедиться, что векторы $e_{1} v$ и $\left(e_{1}^{2}-\frac{2}{3} e_{2}\right) v$ являются особыми в модуле $V(0,0)$ с градуировками 1 и 2 соответственно.

Включения подмодулей $V\left(\frac{3 k^{2} \pm k}{2}\right)$ дают нам точную последовательность ([19], [11], [12])

$$
\begin{gathered}
\cdots \longrightarrow V\left(\frac{3(k+1)^{2}-(k+1)}{2}, 0\right) \oplus V\left(\frac{3(k+1)^{2}+(k+1)}{2}, 0\right) \stackrel{\delta_{k+1}}{\longrightarrow} V\left(\frac{3 k^{2}-k}{2}, 0\right) \oplus V\left(\frac{3 k^{2}+k}{2}, 0\right) \longrightarrow \\
\quad \ldots \stackrel{\delta_{3}}{\longrightarrow} V(5,0) \oplus V(7,0) \stackrel{\delta_{2}}{\longrightarrow} V(1,0) \oplus V(2,0) \stackrel{\delta_{1}}{\longrightarrow} V(0,0) \stackrel{\varepsilon}{\longrightarrow} \mathbb{C} \longrightarrow 0,
\end{gathered}
$$

где $\delta_{k}$ определены с помощью операторов $S_{p, q} \in U\left(L_{1}\right)$ :

$$
\begin{aligned}
\delta_{k+1}\left(\begin{array}{l}
x \\
y
\end{array}\right)= & \left(\begin{array}{cc}
S_{1,3 k+1} & S_{2 k+1,2} \\
-S_{2 k+1,1} & -S_{1,3 k+2}
\end{array}\right)\left(\begin{array}{l}
x \\
y
\end{array}\right), \quad k \geqslant 1, \\
& \delta_{1}\left(\begin{array}{l}
x \\
y
\end{array}\right)=\left(S_{1,1}, S_{1,2}\right)\left(\begin{array}{l}
x \\
y
\end{array}\right),
\end{aligned}
$$

a $\varepsilon$ - проекция на одномерный $\mathbb{C}$-подмодуль, порожденный вектором $v$.

Теорема 4.5 ([19], [11]). Точная последовательность (19), рассматриваемая как последовательность $L_{1}$-модулей, является свободной резольвентой одномерного тривиального $L_{1}$-модуля $\mathbb{C}$.

Следствие 4.6 [11]. Пусть $V$ есть $L_{1}$-модуль. Тогда когомологии $H^{*}\left(L_{1}, V\right)$ изоморфны когомологиям комплекса

$$
\ldots \stackrel{d_{k+1}}{\longleftarrow} V \oplus V \stackrel{d_{k}}{\longleftarrow} V \oplus V \stackrel{d_{k-1}}{\longleftarrow} \ldots \stackrel{d_{1}}{\longleftarrow} V \oplus V \stackrel{d_{0}}{\longleftarrow} V
$$


с дифберенииалами, задаваемыми формулой

$$
\begin{gathered}
d_{k}\left(\begin{array}{l}
m_{1} \\
m_{2}
\end{array}\right)=\left(\begin{array}{ll}
S_{1,3 k+1} & -S_{2 k+1,1} \\
S_{2 k+1,2} & -S_{1,3 k+2}
\end{array}\right)\left(\begin{array}{c}
m_{1} \\
m_{2}
\end{array}\right), \quad k \geqslant 1, \\
d_{0}(m)=\left(\begin{array}{c}
S_{1,1} m \\
S_{1,2} m
\end{array}\right), \quad m, m_{1}, m_{2} \in V .
\end{gathered}
$$

Далее мы будем рассматривать градуированные модули $V=\bigoplus_{i} V_{i}$ только с одномерными однородными компонентами $V_{i}$. Задавать их будем при помощи специального базиса $f_{i}, V_{i}=\left\langle f_{i}\right\rangle(j \in \mathbb{Z}$ в бесконечномерном случае и $j \in \mathbb{Z}$, $m \leqslant j \leqslant n$ в конечномерном). Для заданного градуированного $L_{1}$-модуля $V=$ $\bigoplus_{i} V_{i}$ введем числа $\sigma_{p, q}(j) \in \mathbb{K}$, такие, что

$$
S_{p, q} f_{j}=\sigma_{p, q}(j) f_{j+p q} .
$$

Пример 4.7. Хорошо известный $L_{1}$-модуль $F_{\lambda, \mu}$ тензорных плотностей [14]:

$$
e_{i} f_{j}=(j+\mu-\lambda(i+1)) f_{i+j} \quad \forall i \in \mathbb{N}, j \in \mathbb{Z},
$$

где $\lambda, \mu$ - два комплексных параметра.

Вспомнив конструкцию $L_{1}$-модуля $\widetilde{V}_{\text {gr }}$ из теоремы 3.1 , можно неформально сказать, что он «склеен» из двух модулей $F_{-1,1}$ и $F_{0,0}$.

Следствие 4.8 [11]. Пусть $V=\bigoplus_{i} V_{i}-$ градуированный $L_{1}$-модуль над полем $\mathbb{K}=\mathbb{R}$ или $\mathbb{C}$. Тогда однородные когомологии $H_{s}^{*}\left(L_{1}, V\right)$ изоморфнь когомологиям следующего комплекса:

$$
\cdots \stackrel{D_{k+1}}{\longleftarrow} \mathbb{K} \oplus \mathbb{K} \stackrel{D_{k}}{\longleftarrow} \mathbb{K} \oplus \mathbb{K} \stackrel{D_{k-1}}{\longleftarrow} \cdots \stackrel{D_{1}}{\longleftarrow} \mathbb{K} \oplus \mathbb{K} \stackrel{D_{0}}{\longleftarrow} \mathbb{K},
$$

где дифференииаль $D_{k}$ задаются числовыми матрицами

$D_{k}=\left(\begin{array}{ll}\sigma_{1,3 k+1}\left(s+\frac{3 k^{2}-k}{2}\right) & -\sigma_{2 k+1,1}\left(s+\frac{3 k^{2}-k}{2}\right) \\ \sigma_{2 k+1,2}\left(s+\frac{3 k^{2}+k}{2}\right) & -\sigma_{1,3 k+2}\left(s+\frac{3 k^{2}+k}{2}\right)\end{array}\right), \quad k \geqslant 1, \quad D_{0}=\left(\begin{array}{l}\sigma_{1,1}(s) \\ \sigma_{1,2}(s)\end{array}\right)$.

\section{§5. Формулы Бенуа-Сент-Обана и дифференциалы спектральной последовательности}

В этом параграфе мы будем считать когомологии $H^{*}\left(L_{1}, \widetilde{V}_{\mathrm{gr}}^{m, n}\right)$ при помощи резольвенты Фейгина-Фукса-Роча-Карриди-Уоллаха, построение которой мы напомнили в §4. Для конкретных вычислений необходимы явные формулы для дифференциалов (22) или (24). В свое время Фейгин и Фукс вычислили ([11], [13]) матричные элементы $\sigma_{p, q}(j)$ дифференциалов $(24)$ для двух важных примеров градуированных $L_{1}$-модулей, но общей формулы для операторов $S_{p, q}(t)$ так и не нашли. В конце 80-х годов Бенуа и Сент-Обан нашли красивое выражение для $S_{p, 1}(t)$.

Теорема 5.1 ([5], см. полное доказательство в [3]).

$$
S_{p, 1}(t)=\sum_{\substack{i_{1}, \ldots, i_{s} \\ i_{1}+\cdots+i_{s}=p}} c_{p}\left(i_{1}, \ldots, i_{s}\right) t^{p-s} e_{i_{1}} \cdots e_{i_{s}}
$$


где суммирование происходит по всем неупорядоченным разбиениям натурального числа $p$. Коэффичиенты $c_{r}\left(i_{1}, \ldots, i_{s}\right)$ определены формулами

$$
c_{r}\left(i_{1}, \ldots, i_{s}\right)=\prod_{\substack{1 \leqslant k<r \\ k \neq i_{1}+\cdots+i_{l}, l=1, \ldots, s-1}} k(r-k) .
$$

\section{Пример 5.2 .}

$S_{1,1}(t)=e_{1}, \quad S_{2,1}(t)=e_{1}^{2}+t e_{2}, \quad S_{3,1}(t)=e_{1}^{3}+t\left(2 e_{1} e_{2}+2 e_{2} e_{1}\right)+4 t^{2} e_{3}$.

Формулы (25) будет вполне достаточно для наших целей. Вычислим элементы $\sigma_{p q}(j)$ дифференциалов $(24)$ для модуля $\widetilde{V}_{\mathrm{gr}}^{m, n}$ с базисом $f_{j}$. Простое вычисление показывает, что

$$
S_{3,1}(t) f_{-2}=\left(-2 t+4 t^{2}\right) f_{1}=4 t\left(t-\frac{1}{2}\right) f_{1}=(2 !)^{2} \prod_{i=1}^{2}\left(t+\frac{i-2}{i(3-i)}\right) f_{1} .
$$

Лемма 5.3. Пусть $j<0 u p+j>0$. Тогда

$$
S_{p, 1}(t) f_{j}=(p-1) !^{2} \prod_{i=1}^{p-1}\left(t+\frac{i+j}{i(p-i)}\right) f_{j+p} .
$$

Доказательство. Следующее предложение проверяется непосредственно.

Предложение 5.4. Пусть $j<0 u i_{1}+\cdots+i_{s}+j>0$ при положительных $i_{1}, \ldots, i_{s}$. Тогда

$$
e_{i_{1}} \cdots e_{i_{s-1}} e_{i_{s}} f_{j}=\prod_{l=2}^{s}\left(i_{l}+\cdots+i_{s}+j\right) f_{i_{1}+\cdots+i_{s}+j}
$$

Формулу $(26)$ для коэффициента $c_{p}\left(i_{1}, \ldots, i_{s}\right)$ можно записать в виде

$$
\begin{aligned}
c_{p}\left(i_{1}, \ldots, i_{s}\right) & \\
& =\frac{(p-1) !^{2}}{i_{1}\left(i_{1}+i_{2}\right) \cdots\left(i_{1}+\cdots+i_{s-1}\right)\left(p-i_{1}\right)\left(p-i_{1}-i_{2}\right) \cdots\left(p-i_{1}-\cdots-i_{s-1}\right)} .
\end{aligned}
$$

Воспользуемся формулой Бенуа-Сент-Обана (25) для вычисления значения $S_{p, 1}(t) f_{j}$, переходя к новым индексам суммирования $k_{1}=i_{1}, k_{2}=i_{1}+i_{2}, \ldots$, $k_{s-1}=i_{1}+i_{2}+\cdots+i_{s-1}$ :

$$
\begin{aligned}
& S_{p, 1}(t) f_{j}=\sum_{\substack{\left(i_{1}, \ldots, i_{s}\right) \in \mathbb{N}^{s} \\
i_{1}+\cdots+i_{s}=p}} \frac{t^{p-s}(p-1) !^{2}\left(i_{s}+j\right) \cdots\left(i_{s}+\cdots+i_{2}+j\right)}{i_{1} \cdots\left(i_{1}+\cdots+i_{s-1}\right)\left(p-i_{1}\right) \cdots\left(p-i_{1}-\cdots-i_{s-1}\right)} f_{j+p} \\
& =(p-1) !^{2} \sum_{1 \leqslant k_{1}<\cdots<k_{s-1} \leqslant p-1} t^{p-s} \frac{\left(k_{1}+j\right)}{k_{1}\left(p-k_{1}\right)} \cdots \frac{\left(k_{s-1}+j\right)}{k_{s-1}\left(p-k_{s-1}\right)} f_{j+p} \\
& =(p-1) !^{2} \prod_{i=1}^{p-1}\left(t+\frac{i+j}{i(p-i)}\right) f_{j+p}=F_{j, p}(t) f_{j+p},
\end{aligned}
$$

где $F_{j, p}(t)=(p-1) !^{2} \prod_{i=1}^{p-1}\left(t+\frac{i+j}{i(p-i)}\right)$. 
Предложение 5.5. Пусть $j<0 u p \geqslant 2$. Тогда

$$
F_{j, p}(-3 / 2) \neq 0, F_{j, p}(-2 / 3)=0 \Longleftrightarrow p+3 j=1 .
$$

Доказательство очевидно, поскольку $-3 / 2$ не является корнем многочлена $F_{j, p}(t)$, а $-2 / 3-$ один из его корней тогда и только тогда, когда $p+3 j=1$.

Завершим доказательство основной теоремы. Для этого рассмотрим комплекс (23), вычисляющий однородные когомологии $H_{s}^{*}\left(L_{1}, \widetilde{V}_{\mathrm{gr}}^{m, n}\right)$, в следующих двух случаях

1) $s=-m-1-\frac{3 k^{2}+k}{2}$ и $m+n=2 k-1$;

2) $s=-\frac{3(k+1)^{2}-(k+1)}{2}, m=2 k, n=k+1$.

В первом случае в комплексе (23) имеется лишь один дифференциал, который может быть нетривиальным:

$$
D_{k}=\left(\begin{array}{cc}
0 & -\sigma_{2 k+1,1}(-m-1) \\
0 & 0
\end{array}\right) .
$$

Из леммы 5.3 и предложения 5.5 следует, что

$$
\sigma_{2 k+1,1}(-m-1)=F_{-m-1,2 k+1}(-3 / 2) \neq 0 .
$$

Это эквивалентно нетривиальности дифференциала $d_{2 k+1}$ из леммы 3.4. Эту эквивалентность мы устанавливаем по следующему соображению: имеется два способа подсчета когомологий $\left.H_{-m-1+\left(3 k^{2}+k\right) / 2}^{k}\left(L_{1}, V_{\mathrm{gr}}^{m, n}\right)\right)$, при помощи спектральной последовательности $(*)$ и при помощи резольвенты. Если, например, $d_{2 k+1}=0$, а $D_{k} \neq 0$, то у нас получатся просто разные ответы, чего не может быть. Несомненно, будет полезно установить явную связь дифференциалов $d_{2 k+1}$ и $D_{k}$, исходя из общей гомологической алгебры.

Во втором случае в (23) нетривиальным может быть также только один дифференциал $D_{k}$ :

$$
D_{k}=\left(\begin{array}{cc}
0 & -\sigma_{2 k+1,1}(-2 k-1) \\
0 & -\sigma_{1,3 k+2}(-2 k-1)
\end{array}\right) .
$$

Далее имеем

$$
S_{2 k+1,1}(-3 / 2) f_{-2 k-1}=\sigma_{2 k+1,1}(-2 k-1) f_{0}=F_{-2 k-1,2 k+1}(-2 k-1)(-3 / 2) f_{0},
$$

что равно нулю, согласно предложению 5.5. При этом

$$
\sigma_{1,3 k+2}(-2 k-1) f_{k+1}=S_{3 k+2,1}(-2 / 3) f_{-2 k-1}=F_{-2 k-1,3 k+2}(-2 / 3) f_{k+1} \neq 0 .
$$

Значит, дифференциал $d_{3 k+2}$ из леммы 3.6 является нетривиальным. Аргументы те же, что и в предыдущем случае. Доказательство теоремы 3.1 окончено.

\section{ЛитератУра}

[1] И. В. Артельных, Произведения Масси и спектральная последовательность Бухштабера, УМН, 55:3 (2000), 165-166.

[2] И. К. Бабенко, И. А. Тайманов, Произведения Масси в симплектических многообразиях, Матем. сб., 191:8 (2000), 3-44.

[3] M. Bauer, Ph. Di Francesco, C. Itzykson, J.-B. Zuber, Covariant differential equations and singular vectors in Virasoro representations, Nuclear Phys. B, 362:3 (1991), 515562.

[4] Y. Benoist, Une nilvariété non affine, J. Differential Geom., 41:1 (1995), 21-52. 
[5] L. Benoit, Y. Saint-Aubin, Degenerate conformal field theories and explicit expressions for some null vectors, Phys. Lett. B, 215:3 (1988), 517-522.

[6] В. М. Бухштабер, Характер Чженя-Долъда в кобордизмах. I, Матем. сб., 83(125):4(12) (1970), 575-595.

[7] В. М. Бухштабер, А. В. Шокуров, Алгебра Ландвебера-Новикова и формалъные векторные поля на прямой, Функц. анализ и его прил., 12:3 (1978), 1-11.

[8] I. M. Gelfand, The cohomology of infinite dimensional Lie algebras: some questions of integral geometry, in: Actes du Congres International des Mathématiciens (Nice, 1970), t. 1, Gauthier-Villars, Paris, 1971, 95-111.

[9] Л. В. Гончарова, Когомологии алгебр Ли формалъных векторных полей на прямой, Функц. анализ и его прил., 7:2 (1973), 6-14; 7:3 (1973), 33-44.

[10] Б. Л. Фейгин, Д. Б. Фукс, Кососимметрические инвариантные дифференииальные операторы на прямой и модули Верма над алгеброй Вирасоро, Функц. анализ и его прил., 16:2 (1982), 47-63.

[11] B. L. Feigin, D. B. Fuchs, Verma modules over Virasoro algebra, in: Lecture Notes in Math., vol. 1060, Springer-Verlag, Berlin, 1984, 230-245.

[12] B. L. Feigin, D. B. Fuchs, Representations of the Virasoro algebra, in: Representations of Lie Groups and Related Topics, Adv. Stud. Contemp. Math., vol. 7, Gordon \& Breach, New York, 1990, 465-554.

[13] B. L. Feigin, D. B. Fuchs, V. S. Retakh, Massey operations in the cohomology of the infinite dimensional Lie algebra $L_{1}$, in: Lecture Notes in Math., vol. 1346, SpringerVerlag, Berlin, 1988, 13-31.

[14] Д. Б. Фукс, Когомологии бесконечномерных алгебр Ли, Наука, М., 1984.

[15] D. B. Fuchs, Singular vectors over the Virasoro algebra and extended Verma modules, в кн.: Adv. Soviet Math., т. 17, Amer. Math. Soc., Providence, RI, 1993, 65-74.

[16] V. G. Kac, Contravariant form for infinite-dimensional Lie algebras, in: Lecture Notes in Phys. B, vol. 94, 1979, 441-445.

[17] D. Kraines, Massey higher products, Trans. Amer. Math. Soc., 124 (1966), 431-449.

[18] W. S. Massey, Some higher order cohomology operations, in: Simposium International de Topologia Algebraica, La Universidad National Autónoma de Mexico and UNESCO, Mexico City, 1958, 145-154.

[19] A. Rocha-Carridi, N. R. Wallach, Characters of irreducuble representations of the Lie algebra of vector fields on the circle, Invent. Math., 72:1 (1983), 57-75.

Московский государственный университет

Поступило в редакцию им. М. В. Ломоносова, 10 марта 2009 г.

механико-математический факультет

e-mail: million@mech.math.msu.su 